\title{
Body Mass Index and Western Ontario \& McMaster Universities Osteoarthritis Index in Patients with Knee Osteoarthritis in Dr. Hasan Sadikin General Hospital, Bandung in November 2012
}

\author{
Ainna Binti Mohamad Dat, ${ }^{1}$ Tertianto Prabowo, ${ }^{2}$ Alwin Tahid ${ }^{3}$ \\ ${ }^{1}$ Faculty of Medicine Universitas Padjadjaran, ${ }^{2}$ Department of Physical Medicine and \\ Rehabilitation Faculty of Medicine, Universitas Padjadjaran/Dr. Hasan Sadikin General Hospital \\ Bandung, ${ }^{3}$ Department of Anatomy and Cell Biology Faculty of Medicine Universitas Padjadjaran
}

\begin{abstract}
Background: Osteoarthritis is one of the major disabilities among elderly. One of its well-recognized potent risk factors is obesity. The aim of this study was to identify the body mass index and severity of knee osteoarthritis patients who were treated in Dr. Hasan Sadikin General Hospital Bandung.

Methods: A descriptive study was carried out to 9 patients of the Medical Rehabilitation Policlinic at Dr. Hasan Sadikin General Hospital Bandung in November 2012. Patients were diagnosed as having knee Osteoarthritis based on American College of Rheumatology clinical classification. Exclusion criteria were patient having previous trauma in spine and lower limb, having bleeding disorder like hemophilia, incomplete data in medical records and incomplete data in questionnaire. Western Ontario and McMaster Universities Osteoarthritis Index (WOMAC) was used to measure the intensity of pain, stiffness, and functional difficulty. The weight $(\mathrm{kg})$ and height $(\mathrm{cm})$ of the patients were measured and the Body Mass Index was calculated by Weight $(\mathrm{kg}) /$ Height $^{2}(\mathrm{~m})$. The data were analyzed using frequency distribution.

Results: The patients who came to the Medical Rehabilitation Policlinic had ranged in age from 57 to78 years, mostly female with knee Osteoarthritis bilateral. Out of 9 patients, 5 patients were overweight, followed by normal BMI and obese type I. Patient with obese type 1 had the highest WOMAC score.

Conclusions: Most of the patients with knee osteoarthritis bilateral are overweight and the patient with obese type 1 has the highest WOMAC score. [AMJ.2015;2(3):453-7]
\end{abstract}

Keywords: Body mass index, osteoarthritis, WOMAC score

\section{Introduction}

In developed countries, Osteoarthritis is one of the ten most disabling diseases commonly affecting the elderly and one of the major causes of disability among them. The incidence of knee Osteoarthritis is 240 in 100 $0000^{1,3}$ It is defined as degeneration of articular cartilage, hypertrophy of bone at the margins, and changes in the synovial fluid, making it as the non-inflammatory degenerative joint disease. ${ }^{4}$ Cervical and lumbosacral spine, hip, knee, first metatarsal phalangeal joint, and interphalangeal joint are the commonly affected joints and it might also spread to the wrist, elbow and ankle. ${ }^{2}$

Obesity is one of the well-recognized potent risk factors. This is because during single leg stance, three to six times body weight will be exerted across the knee. Thus, in the overweight patient, it may cause an increased load in the axial loading at the knee joint due to repetitive application, leading to degeneration of articular cartilage and sclerosis at the subchondral bone..$^{2,5}$

Classification of obesity is made based on Body Mass Index (BMI) classification. It is a simple index of weight-to-height and can be calculated by dividing weight in kilograms with the square of the height in meters (kg/ $\mathrm{m} 2$ ). Therefore, if the patient's BMI is 30.00 or more, it is already considered as obese. ${ }^{6}$

In assessing patient with chronic illness, their perceptions of their health status are important since the therapeutic goals include preserving and optimizing their

Correspondence: Ainna Binti Mohamad Dat, Faculty of Medicine, Universitas Padjadjaran, Jalan Raya BandungSumedang Km.21, Jatinangor, Sumedang, Indonesia, Phone: +6287827935036 Email: ainna.rockafella@gmail.com 
Table 1 BMI Classification ${ }^{6}$

\begin{tabular}{lcc}
\hline & Classification & BMI $\left(\mathbf{k g} / \mathbf{m}^{2} \mathbf{~}\right.$ \\
\hline Underweight & $<18.50$ \\
Normal & $18.50-24.00$ \\
Overweight & $25.00-29.99$ \\
Obese Class 1 & $30.00-34.99$ \\
Obese class II & $35.00-39.99$ \\
Obese class III & $>40.00$ \\
\hline
\end{tabular}

health-related quality of life. ${ }^{7}$ Therefore, to evaluate the severity of knee Osteoarthritis, Western Ontario and McMaster Universities Osteoarthritis Index (WOMAC) was being used since it has the ability to assess the pain, stiffness, and physical function of the patient. ${ }^{8}$

The aim of the study was to identify the Body Mass Index and severity of knee Osteoarthritis patients who came to Medical Rehabilitation Policlinic at Dr. Hasan Sadikin General Hospital Bandung.

\section{Methods}

A descriptive study was carried out to 9 patients at the Medical Rehabilitation Policlinic of Dr. Hasan Sadikin General Hospital Bandung in November 2012. Patients were diagnosed as having knee Osteoarthritis based on American College of Rheumatology clinical classification. To be included in this classification, at least 3 of the following symptoms occurred; aged of 50 years old or more, with stiffness, crepitus, bony tenderness, bony enlargement, and no palpable warmth. Exclusion criteria were the patient having previous trauma in spine and lower limb, patient having bleeding disorder like hemophilia, incomplete data in medical records, and incomplete data in questionnaire. All patients were given informed consent before participating in this study.
For evaluating the characteristics of severity, the pain and self-report function was observed, whereby WOMAC will be used since it is a scale to measure the intensity of pain, stiffness, and functional difficulty in people with knee or hip OA. ${ }^{9}$ It consists of 3 subscales with 4 items total (5 pain, 2 stiffness and 17 physical function). ${ }^{10}$ There are 5 response options offered from Likert version ranging from 'none' which is scored as 0 , 'mild' as 1 , 'moderate' as 2, 'severe' as 3 and 'extreme' as $4 .{ }^{10}$ The scores are summed for each section to produce pain, stiffness, and physical function subscale score.10 Thus, the subscale score for pain can vary from $0-20$, subscale score for stiffness can vary from $0-8$, and lastly subscale score for physical function can vary from 0-68.10 The total WOMAC score range from 0-96 where 0 represents the best health status and 96 represents the worst health possible status.11 The time taken to administer the test is 5 minutes. ${ }^{10}$

To calculate the BMI, the weight $(\mathrm{kg})$ and height $(\mathrm{cm})$ of the patient will be taken. The patient will be wearing light clothing, and shoes will be taken off when measuring the weight and height. Weight will be measured to the nearest 0.1 kilogram using an electronic scale while standing. Height will be measured to the nearest 0.01 meter .To calculate the BMI, the formula is:

Table 2 Characteristics of BMI

\begin{tabular}{|c|c|}
\hline BMI Category & Frequency \\
\hline Underweight & 0 \\
\hline Normal & 3 \\
\hline Overweight & 5 \\
\hline Obese 1 & 1 \\
\hline Obese 2 & 0 \\
\hline Total & 9 \\
\hline
\end{tabular}




$$
\mathrm{BMI}=\frac{\text { Weight }(\mathrm{kg})}{\operatorname{Height}^{2}(\mathrm{~m})}
$$

The classification of underweight, normal, overweight and obesity is based on the World Health Organization (WHO) recommendation (Table 1).

\section{Results}

The patients who came to the Medical Rehabilitation Policlinic were 57-78 years old, mostly female with knee Osteoarthritis Genu bilateral. From 9 patients participating in this study, 5 patients were overweight, followed by normal BMI, and obese type I.

The diagram shows that patients whose BMI in obese 1 category had highest mean of WOMAC index score, indicating the highest severity. Consecutively, the diagram shows findings followed by normal BMI patient and overweight BMI patient.

\section{Discussion}

The patients who came were in age range of 57-78 years old. Older age may increase the prevalence rates of $30-50 \%$ of the adults whose age were more than 65 years with Osteoarthritis for all joints. This is one of the strongest determinants in Osteoarthritis.,12 Body Mass Index (BMI), age, and inability to maintain balance are reported to have positive relation to these characteristics..$^{5}$ Joint vulnerability will increase with age because the cartilage matrix in young cartilage will be stimulated by chondrocytes due to dynamic loading of joints. However, in aged cartilage, it will be less responsive to these stimuli. ${ }^{2}$

Meanwhile in gender, most patients who came to the Medical Rehabilitation Policlinic at Dr. Hasan Sadikin General Hospital Bandung were female. Since women undergo menopause, it may contribute to the risk of hormonal loss which causes an increasing in joint vulnerability. ${ }^{2}$ In addition to that, the respondents with previous occupation history make up the highest frequency due to their daily activities that require them to move around actively. This biomechanical factor may cause the muscle exhausted gradually due to long days at work, which in turn may reduce the effectiveness in joint protector. ${ }^{2}$

Most respondents came with knee Osteoarthritis Genu bilateral, whereby most of them having other accompanying diseases. Instability, varus or valgus misalignment, chondrocalcinosis, and low intake of Vitamin $\mathrm{C}$ and Vitamin D are some of the risk factors that may progress to the severity of knee Osteoarthritis, which present in these accompanying diseases. ${ }^{13}$

Overweight patients are the highest at risk compared to the other BMI index patients coming to the Medical Rehabilitation Policlinic. The disease progression in those who already have knee Osteoarthritis may be influenced by being heavy, since a person's weight may influence the incidence of Osteoarthritis. ${ }^{5}$ During walking, the person with increasing weight receives excessive forces across the knee. ${ }^{2}$ Thus, this in turn may lead to severity

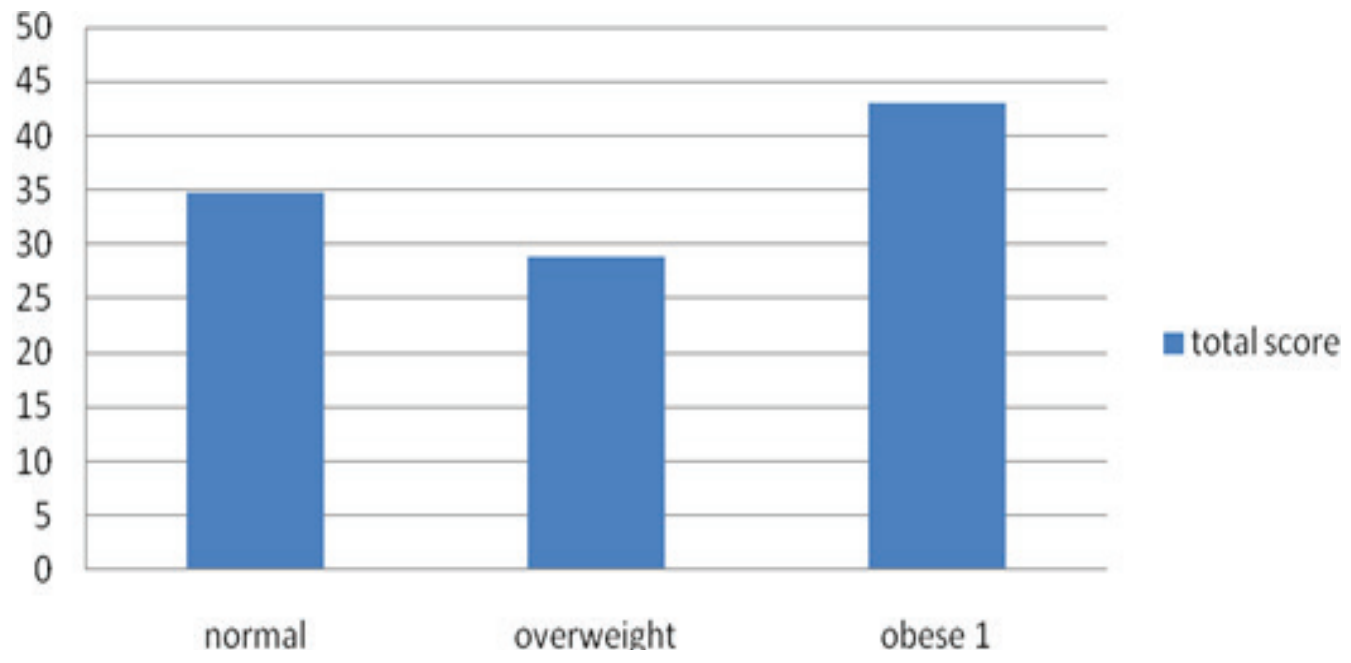

Figure 1 The Characteristics of BMI and Mean of WOMAC Score Index 
progression of knee Osteoarthritis.

The obese type 1 patient has the highest mean total of WOMAC index score. This is because the development of the disease is preceded by the obesity and is not only due to inactivity present to those with the disease. Overweight patients with increased load in weight-bearing joints will mediate the obesity effect on the development and progression of the disease. Therefore obese type 1 patients show higher WOMAC score compared with others. It indicates that obese type 1 has severe knee Osteoarthritis compared with normal and overweight patients.

Meanwhile, comparing normal and overweight patients, the normal BMI patients have higher mean of the total WOMAC index score. This factor could probably occur due to one of the two normal patients is male. As reported from the previous study that it may affect the ability to balance, the severity of knee Osteoarthritis is greater in men compared to that in women. ${ }^{5}$

In other perspective, the diagram shows that weight isnot the only factor that may influence the WOMAC score, other factors may too. Functional and pain related scores can also be influenced by low back pain; such as rising from a chair, getting out of bed, and more. Thus, WOMAC result cannot be indicated to reflect the lower extremity disease only. In addition, the patient may have referred pain whereby the pain is due to hip Osteoarthritis. However, he/she may refer it to the knee. ${ }^{14}$ Besides that, psychological factors also affect the WOMAC scores; such as fatigue and depression. These elements may also lead to actual pain and dysfunction, as reported by the respondents. ${ }^{15}$

The limitation of this study was the method used, which was descriptive study. This was chosen due to time restriction. Therefore, to obtain correlation, the study method that should be used was cohort or case-control.

Besides that, the samples obtained were very small due to time restriction. Apart from that, the variables in this study were incomplete; such as the period of having knee Osteoarthritis, the frequency of visiting medical rehabilitation policlinic, and medicine that had been taken to treat the symptoms of knee Osteoarthritis disease.

In addition, not all confounding factor can be avoided in this study; such as the treatment received by the patient to treat the disease, other accompanying disease, the psychology of the patient, and social-economic status of the patient. This may occur because most of the patients came with severe knee Osteoarthritis.
They could have already had the risk factors causing the knee Osteoarthritis or other progressive factors other the BMI itself. These particular factors may give impact on the knee Osteoarthritis severity.

Understanding further management taken by the patients to overcome the symptom, the treatments should be studied meticulously so that education on proper management to the patient can be done properly in the future. Besides that, obesity without any accompanying disease should be studied in order to contribute to the development or progression of knee Osteoarthritis. As a result, the management of knee Osteoarthritis can be more definite. Eventually, a different type of questionnaire should be used to assess the severity of knee Osteoarthritis other than WOMAC Index Score.

As a conclusion, the characteristics of the BMI in the patients with knee Osteoarthritis who came to the Medical Rehabilitation policlinic at Dr. Hasan Sadikin General Hospital Bandung were mostly overweight. In addition, obese type 1 patients had the highest WOMAC score among the other type of BMI index.

\section{References}

1. WHO. Chronic disease and health promotion, chronic rheumatic condition. 2012 [cited 2012 August 9]. Available from: http://www.who.int/chp/topics/ rheumatic/en/.

2. Fauci AS, Weiner C, Braunwald E, Kasper DL, Hauser SL, Longo DL, et al. Harrison's Value Pack. 17th ed. New York: McGrawHill; 2008.

3. CDC. Arthritis 2011 [cited 2013 January 27]; Available from: http://www.cdc.gov/ arthritis/basics/osteoarthritis.htm.

4. Dorland, editor. Dorland's pocket medical dictionary. 28th ed. Philadelphia: Elsevier Health Sciences; 2008.

5. Pearson-Coel J. Literature review on the effects of obesity on knee osteoarthritis. Orthop Nurs. 2007;26(5):289-92.

6. WHO. BMI classification [cited 2012 August 10]. Available from: http://apps. who.int/bmi/index.jsp?introPage=intro_3. html.

7. Clark JA, Spiro A 3rd, Fincke G, Miller DR, Kazis LE. Symptoms severity of osteoarhritis of the knee: a patientbased measure developed in the veterans health study. J Gerontol A Biol Sci Med Sci. 1998;53(5):M351-60.

8. American College of Rheumatology. 
Westen Ontario and McMaster Universites Osteoarthritis Index (WOMAC). 2011 [cited 2012 July 10]; Available from: http://www.rheumatology.org/practice/ clinical/clinicianresearchers/outcomesinstrumentation/WOMAC.asp.

9. Harrison AL. The influence of pathology, pain, balance and self-efficacy on Function in women with osteoarthritis of the knee. Phys Ther. 2004;84(9):822-31.

10. Morgan AS, Nordberg HS. Western Ontario McMaster (WOMAC) Osteoarthritis Index. [Report] 2011 [cited 2012 August 9] ; Available from: ptoutcome.com/ WOMAC\%20Publish.doc.

11. Hmamouchi I, Allali F, Tahiri L, Khazzani $\mathrm{H}$, Mansouri LE, Alla SAO, et al. Clinically important improvement in the WOMAC and predictor factors for response to nonspecific non-steroidal antiinflammatory drug in osteoarthritis patient. BMC Res Notes. 2012;5:58.

12. Hunter DJ, editor. Osteoarthritis: an issue of clinics in geriatric medicine. 26th ed. Philadelphia: Elsevier Health Sciencies; 2010.

13. Doherty M. Risk factors for progression of knee osteoarthritis. Lancet. 2001;358(9284):775-6.

14. Brandt KD. Diagnosis and nonsurgical management of osteoarthritis. New York: Professional Communications, Incorporated; 2010.

15. Wolfe F. Determinants of WOMAC function, pain and stiffness scores: evidence for the role of low back pain symptoms counts fatigue and depression in osteoarthrits, rheumatoid arthritis and fibromyalgia. Rheumatology (Oxford). 1999;38(4):35561. 